\author{
I.W.A. Browne \\ Nuffield Radio Astronomy Laboratories, \\ Jodre11 Bank.
}

What does intermediate (arcsecond) scale structure tell us about the activity deep in the VLBI cores of galaxies and quasars? There are certain key areas where a knowledge of this structure is particularly relevant. One is in defining the relationships between the various classes of objects leading to unified schemes. A second is in the understanding of jet physics. For the latter it is very important to know if the material in the jets supplied from the nucleus is heavy or light, slow or fast, ejected one side at a time or symmetrically. The optimum resolution to study jet material is on the arcsecond (三 kiloparsec) scale where very high dynamic range maps are possible and where it becomes feasible to find out something about the physical conditions in the surrounding medium from optical and X-ray observations.

\title{
UNIFIED SCHEMES
}

The justification for talking about unified schemes is that they simplify things and that they make testable predictions about a wide range of phenomena. Also one of the important inputs which prompted the idea of a unified scheme came from the study of the intermediate scale structure of compact VLBI sources. The basic proposition of such schemes is that the only difference between a flat and a steep spectrum source is one of aspect; projection and the Doppler boosting turn an extended double source into a compact core-dominated one.

Evidence that there exists some kind of unity between flat and steep radio spectrum quasars comes from several directions and has been discussed in some detail by Orr \& Browne (1982). The situation concerning radio galaxies is not nearly so well defined. Only in one, 3C120, is there direct evidence of relativistic motion. In addition flat spectrum radio galaxies are rare, and most of these when studied optically, turn out to be BL Lac objects (e.g. Ap.Lib and 3C371). This latter point leads me to propose a 'unification' between elliptical radio galaxies and BL Lac objects.

+ Discussion on nage 415 
If we assume that the characteristic optical continum radiation from a BL Lac object is relativistically beamed there must be a large 'parent population' not showing BL Lac behaviour. Such a parent population would have the following properties:-

1) be extended radio sources, because BL Lacs themselves have diffuse radio emission (Stannard \& McIlwrath 1982; Ulvestad et a1. 1983)

2) be elliptical galaxies since nearby BL Lac objects a11 lie in elliptical galaxies

3) have very weak or non-existent emission lines

4) have a significantly higher space density than BL Lac objects themselves.

The obvious conclusion is that the BL Lac 'parents' are elliptical radio galaxies. Furthermore, by comparing the local space density of BL Lac objects and elliptical radio galaxies of a given extended radio luminosity we can deduce an upper limit to the average Lorentz factor of the radiating material. The simplest possible situation is that it is only when the angle to the line of sight, $\theta$, is less than $1 / \gamma$ does the object display characteristic BL Lac properties. In this case the proportion of objects expected to be BL Lacs is $[1-\cos (1 / \gamma)]$. Comparing the local space densities of BL Lacs of extended radio luminosity $\geq 10^{22} \mathrm{~W} \mathrm{~Hz}^{-1} \mathrm{sr}^{-1}$, with that of elliptical radio galaxies of the same luminosity, gives $\gamma \lesssim 5.4$ (Browne, 1983).

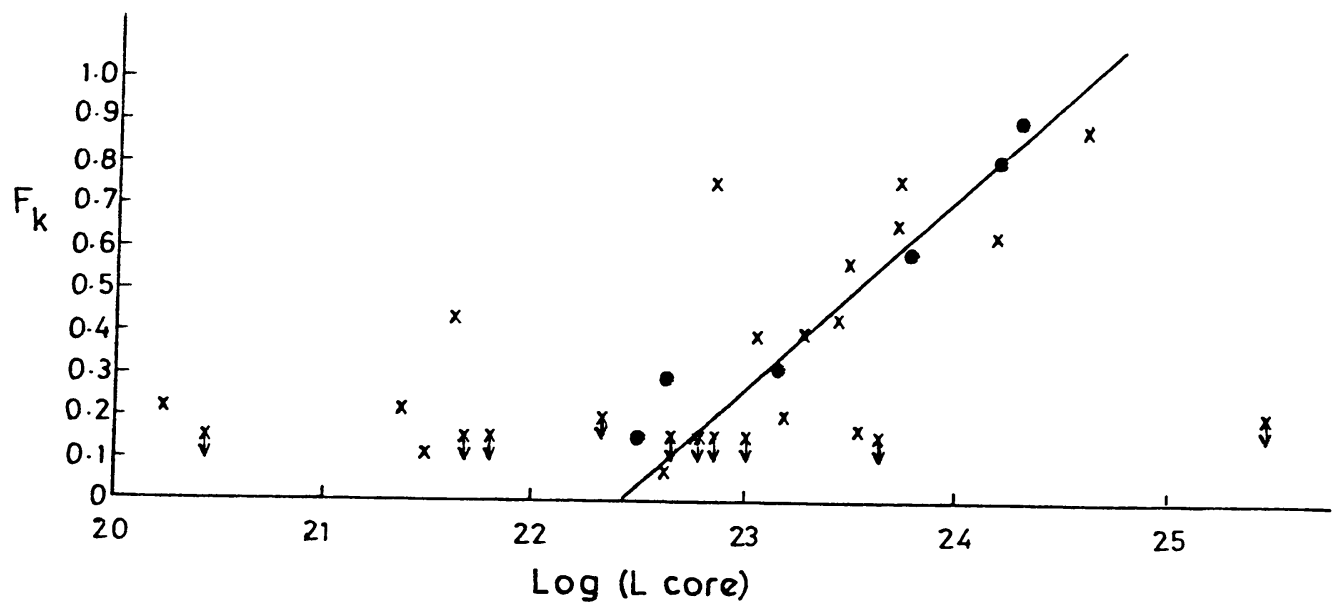

Fig.1 The fraction of galaxy flux in an infrared core as a function or core radio luminosity. $F_{K}$ is the function of $K$ band light in the galaxy core. Lcore is the $\lambda 6 \mathrm{~cm}$ core luminosity in $\mathrm{W} \mathrm{Hz}^{-1} \mathrm{sr}^{-1}$. Crosses denote radio galaxies; filled circles BL Lac objects. Data are taken from Heckman et al. (1983). 
Before leaving the subject of BL Lacs and radio galaxies I would like to mention a consequence of the proposed unification. Such a scheme predicts the existence of optical BL Lac cores in all elliptical radio galaxies. How might we find these? In face-on objects $\left(\theta \sim 90^{\circ}\right)$ the cores would be too weak to detect, but there might be a chance in radio galaxies with fairly strong cores which we might expect to be only at moderate angles to the line-of-sight. Fortunately there exist some observations which at least partially check this prediction. Heckman et al. (1983) have looked for nonstellar optical/infrared nuclear continuum emission from 42 radio galaxies selected by their core strength. They find that most of these objects do have continuum emission and this is roughly proportional to the strength of the compact radio source. Fig.1 shows the fraction of the infrared flux contained in the core plotted against core radio luminosity for Heckman et al's sample. Whenever the core-radio luminosity is sufficient an infrared core is nearly always present. It is also worth pointing out that all objects appear to follow the same trends irrespective of whether they are classified as radio galaxies or BL Lacs. A careful study of all these cores in the optical to see if they have the characteristic variability and high polarization of BL Lacs is clearly a very important investigation.

\section{JET PROPERTIES (ONE-SIDED OR TWO-SIDED; FAST OR SLOW?)}

Do the central objects in radio galaxies and quasars always eject two beams or is the ejection sometimes one-sided? Are the jets relativistic? The study of kiloparsec scale jets offers some useful pointers, but not as yet any definitive answers to these questions. In the following I will try and review some of the evidence, concentrating on ways of estimating jet speeds.

Only if jets are relativistic on all scales can the idea of symmetrical ejection be reconciled with the observational evidence that jets appear very asymmetric. On the VLBI scale direct observation of superluminal behaviour indicates that jets start off relativistically. I will assume that there is relativistic motion in al1 VLBI cores and consider the question of the speed of Kiloparsec jets.

\section{Jet Continuity}

VLBI jets almost always look as if they will join up with the arcsecond jets. Misalignments are common but usually $<90^{\circ}$ and, even in the case of large bends, the sense of curvature of the structure usually suggests continuity (Browne et a1.1983). The obvious interpretation is that Doppler favouritism is responsible for the asymmetry on all scales. However, there is in fact no reason why the jets should not be intrinsically asymmetric, having their asymmetry further enhanced by Doppler boosting.

Could jets be one-sided, start off fast, but slow to subrelativistic 
speed 1ater? To answer this question the relative strengths of VLBI cores + jets and arcsecond jets should be examined for a wide range of angles to the line of sight. If there is slowing one would expect a large dispersion in this ratio. Observationally, when we see an arcsecond jet it is usually associated with a VLBI core of roughly similar flux density. This suggests that there is not much slowing as the jet moves outwards from the nucleus. Moore (1983) has attempted to quantify this argument, by trying to see if there is any evidence for 'disembodied jets' (I.E. strong jets with no cores). From the numbers he concludes that kiloparsec scale jets must be relativistic.

2. Other evidence for jet speeds

a) Precession mode1s

Several groups have tried to model rotationally symmetric sources using ballistic precessing beam models. Gower et al. (1982) have looked at a wide range of sources and find that they can successfully reproduce the main features of the structures with precession models. They usually require mildly relativistic beams. Quasars require the fastest beams.

Muxlow (this Conference) has fitted a similar precession model to the very twisted quasar 3C418. He ends up with a mild preference for a beam with $\mathrm{v} / \mathrm{c}=0.98$.

In contrast Hunstead et a1. (1983) conclude that the beam speeds in the strikingly rotationally symmetric quasar $2300-189$ must be $<0.3 \mathrm{c}$. Their precession model reproduces the ridge line geometry of the quasar very we11, but not the intensity distribution which is much brighter on one side of the core than the other.

\section{b) Bending by Ambient Medium}

Like the results from precession models attempts to deduce jet speeds from modelling jet bends produced by winds or atmospheres, give varying answers. For the large scale bent tails in low luminosity sources, velocities of a few thousand $\mathrm{km} \mathrm{s}^{-}$are preferred. However, for the small scale jets in the nucleus of 3C293 van Breugel et al. (1983) deduce a beam velocity $\gtrsim 4 \times 10^{4} \mathrm{~km} \mathrm{~s}^{-1}$.

To sum up the situation on beam velocities we can say the following:-

1) If beams start out at relativistic velocities they stay relativistic.

2) Relativistic motion is sufficient but not necessary to explain the observed asymmetry.

3) Evidence on beam velocities from modelling bends is inconclusive. 


\section{Jet Symmetry}

So far I have discussed work primarily directed at deducing jet velocities. Is there any way in which the related question of jet symmetry can be addressed? One possibility is to investigate sources which show cores, jets and lobes. On the assumption that lobe emission is unbeamed and the observed jet asymmetry is solely due to Doppler beaming, the lobe to which the jet points should be statistically indistinguishable from the opposite lobe. Saika (1981) has attempted such an investigation for relatively low luminosity radio galaxies and finds that jets tend on average to point to the lobes containing the highest brightness features. Work on quasars (Shone, private communication) shows a similar, but less marked, trend. One additional and potentially very important point is that in the few cases we know of sources with multiple hotspots the jet points to the lobe which contains these hotspots. This evidence all supports the conclusion that there are some intrinsic differences in the beams and that Doppler beaming is not the sole cause of the observed asymmetry. The only other possibility is that some of the high brightness features in lobes may be Doppler boosted.

\section{ACKNOWLEDGEMENTS}

I thank David Shone and Peter Wilkinson for helpful discussions and Tim Heckman for permission to use unpublished data.

\section{REFERENCES}

Browne, I.W.A., 1983. Mon.Not.R.astr.Soc., 204, 23P.

Browne, I.W.A., Charlesworth, M., Muxlow, T. $\overline{\text { W.B. }}$, Tzanetakis, A., \& Wilkinson, P.N. 1983. "Astrophysical Jets", eds. A. Ferrari \& A.G. Pacholczyk, Reidel.

Gower, A.C., Gregory, P.C., Hutchings, J.B. \& Unrah, W., 1982. Ap.J., 262, 478 .

Heckman, T.M., Lebofsky, M.J., Rieke, G.H. \& van Breuge1, W., 1983. preprint.

Hunstead, R.W., Murdoch, H.S., Condon, J.J. \& Phillips, M.M., 1983. Mon.Not.R.astr.Soc., submitted.

Moore, P.K., 1983. Mon.Not.R.astr.Soc., submitted.

Orr, M.J.L. \& Browne, I.W.A., 1982. Mon.Not.R.astr.Soc., 200, 1067.

Saika, D.J., 1981. Mon.Not.R.astr.Soc., 197, 11P.

Stannard, D. \& McIlwrath, B., 1982. Nature, 298, 140.

Ulvestad, J.S., Johnston, K.J. \& Weiler, K.W., 1983. Ap.J., 266, 18. van Breuge1, W., Heckman, T.M., Butcher, H. \& Miley, G.K., $198 \overline{3 .}$ preprint. 